\title{
Administration of heat shock protein 65 inhibits murine melanoma growth in vivo
}

\author{
JIE YANG ${ }^{1,2^{*}}$, YANFEI XIE ${ }^{1,3^{*}}$, HUAQIAN WANG $^{1,4}$, YI YAO $^{1}$, JING HOU $^{1}$, YANJUN MA $^{1,5}$, QING ZHANG $^{2}$, \\ YUN XING $^{1}$, JIE WU ${ }^{1}$, TAIMING LI ${ }^{1}$, JUNNIAN ZHENG ${ }^{2}$, JINGJING LIU ${ }^{1}$ and RONGYUE CAO ${ }^{1}$ \\ ${ }^{1}$ Minigene Pharmacy Laboratory, School of Life Science and Technology, China Pharmaceutical University, Nanjing 210009; \\ ${ }^{2}$ Jiangsu Key Laboratory of Biological Cancer Therapy, Xuzhou Medical College, Xuzhou 221002; \\ ${ }^{3}$ School of Basic Medical College, Jiangxi University of Traditional Chinese Medicine, Nanchang 330004; \\ ${ }^{4}$ Department of Pharmacy Engineering, Faculty of Chemical Engineering and Light Industry, Guangdong University \\ of Technology, Guangzhou 510006; ${ }^{5}$ Forensic Center, Department of Criminal Science and Technology, \\ Nanjing Forest Police College, Nanjing 210046, P.R. China
}

Received March 11, 2012; Accepted July 24, 2012

DOI: $10.3892 / \mathrm{mmr} .2012 .1167$

\begin{abstract}
The association between heat shock protein (HSP) 65 and immune diseases has been investigated for many years. The aim of this study was to explore the antitumor effects and possible antitumor mechanism of HSP65. Mice were immunized with HSP65 via subcutaneous injection. Specific IgG antibodies against HSP65 were detected in the sera of immunized mice by enzyme-linked immunosorbent assay and verified by western blot analysis. HSP65 effectively inhibited the growth of tumors as well as both the protective and therapeutic antitumor immunities in the melanoma tumor models of mice and prolonged the survival of the tumor-bearing mice. Furthermore, HSP65 also attenuated tumor-induced angiogenesis in the intradermal model and pulmonary metastasis in the tail intravenously injected model of mice. It was demonstrated that the administration of HSP65 is able to effectively inhibit the growth, angiogenesis and metastasis of murine melanoma in vivo and provide new prospects for the immunotherapy of melanoma.
\end{abstract}

\section{Introduction}

Melanoma ranks as one of the top 10 new types of cancer diagnoses in American males and females (1). The worldwide incidence rate of melanoma is higher than that of other common cancers and doubles approximately every 10-20 years in the

Correspondence to: $\mathrm{Dr}$ Rongyue Cao, Minigene Pharmacy Laboratory, School of Life Science and Technology, China Pharmaceutical University, 24 Tongjia Xiang, Nanjing 210009, P.R. China

E-mail: minigene1@yahoo.com.cn

*Contributed equally

Key words: heat shock protein 65, melanoma, antitumor effect, angiogenesis, metastasis
Caucasian populations, which has resulted in the greatest ever number of skin cancer-related mortalities (1-3). Furthermore, melanoma with dissemination to distant sites and visceral organs is almost invariably incurable, with a median survival time of 8-9 months and a 3-year survival rate of $10-15 \%$ (4). Therefore, malignant melanoma is considered to be one of the most aggressive cancers and has thus become a substantial clinical challenge. It is imperative to establish novel strategies for the treatment of malignant melanoma that are able to improve the quality of life and survival of patients.

Heat shock proteins (HSPs) are widely distributed in nature and are among the most highly conserved molecules of the biosphere. The concentrations of HSPs in non-stressed cells are low, however they are high in stressed cells. Tumors are highlystressed collections of cells that are able to display or express HSPs on the surface, while normal cells do not (5-8). Recent research has revealed the role of HSPs in cancer (6,9-13). In the presence of HSPs, tumors not only cope with stresses via the cytoprotective activities of chaperones, but thrive with upregulated chaperone expression and activity (13-15). It has been reported that overexpression of HSP60 could be utilized as a clinical assessment index (9-11,14-18).

As a member of the HSP60 family, HSP65 is able to induce humoral and cellular immune responses (16) and plays important roles in antigen presentation and cross-presentation (6,17-19). In this study, we investigated the effects of a HSP65 vaccine on the growth, angiogenesis and metastasis of B16-F10 melanoma cells in tumor-bearing mice. The results demonstrated that the HSP65 vaccine was able to markedly inhibit the growth of B16-F10 melanoma in vivo and prolong the survival of tumor-bearing mice, suggesting that HSP65 has potential clinical significance.

\section{Materials and methods}

Preparation of protein and animals. The HSP65 protein was prepared using the same method as previously described (20). Male C57BL/6 mice, 5-6 weeks of age, were purchased from 
A

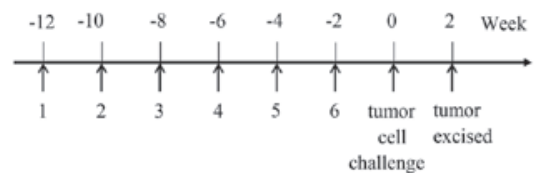

B

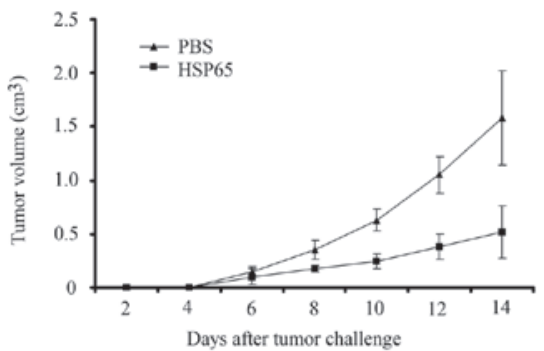

C

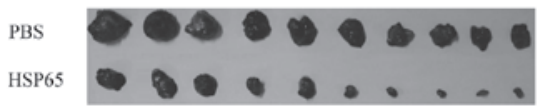

D

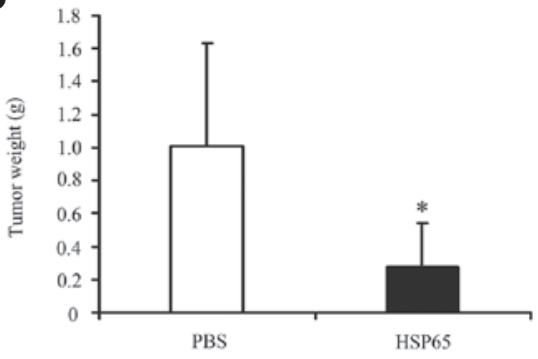

$\mathbf{E}$

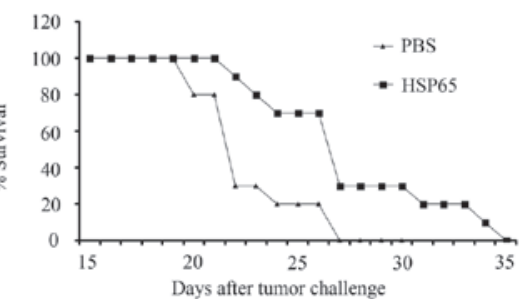

Figure 1. Prophylactic immunization of mice with vaccines. (A) Immunization scheme demonstrated by arrows. (B) In vivo measurement of the tumor growth. (C) Solid tumors were extracted from the mice immunized as described in A. (D) Tumor mass comparison between the mice immunized with PBS and HSP65. ${ }^{*} \mathrm{P}<0.05$. (E) Survival comparison between the mice immunized with HSP65 and PBS. HSP65, heat shock protein 65; PBS, phosphate-buffered saline.

the Laboratory Animal Center of Yangzhou University, China, and housed in plastic cages under pathogen-free conditions on a 12-h light/12-h dark schedule. All experiments were conducted according to the National Institutes of Health Guide for the Care and Use of Laboratory Animals and approved by the Institutional Animal Care and Use Committee of China Pharmaceutical University (IACUC).

Tumor cell lines. The B16-F10 cell line was maintained in our laboratory. Tumor cells were cultured in growth medium containing Dulbecco's modified Eagle's medium (Gibco, Carlsbad, CA, USA) supplemented with $10 \%$ heat-inactivated fetal bovine serum (FBS; Gibco), 2 mmol/1 glutamine, $100 \mathrm{U} / \mathrm{ml}$ penicillin (Gibco) and $100 \mu \mathrm{g} / \mathrm{ml}$ streptomycin at $37^{\circ} \mathrm{C}$ in a humidified atmosphere of $95 \%$ air $/ 5 \% \mathrm{CO}_{2}$.

Immunization and tumor challenge. Male C57BL/6 mice, age 5-6 weeks, were randomly divided into a phosphate-buffered saline (PBS) group and a HSP65 group (20 in each group). The HSP65 group were subcutaneously injected into the left flank with purified protein HSP65 (50 $\mu \mathrm{g}$ in a final volume of $100 \mu \mathrm{l}$ PBS) 6 times at biweekly intervals. Control mice were administered an equal volume of blank PBS. Sera were collected weekly for immunoassay following the initial immunization. Tumor challenge was performed by the subcutaneous injection of murine melanoma cells $\left(5 \times 10^{5}\right.$ cells in $100 \mu \mathrm{l}$ PBS $)$ into the right flank of all mice on the 14th day following the last immunization (Fig. 1A). Tumors were measured in two dimensions using a caliper every 2 days following the tumor challenge (Fig. 1B). The tumor volume was calculated as follows: length $\mathrm{x}$ width ${ }^{2} \mathrm{x} 0.4$ (21). On day 14 following the challenge of tumor cells, half of the mice (10 per group) were sacrificed for tumor weight analysis (Fig. 1C and D). Subsequently, formalin fixation was performed to prepare for hematoxylin and eosin (H\&E) staining and immunohistochemical analysis. The other half of the mice were used for the survival monitoring experiment (Fig. 1E).
Western blot analysis for specificity of anti-HSP65 antibodies. Western blot analysis was used to analyze the specificity of the anti-HSP65 antibodies from the mice immunized with HSP65. The purified protein HSP65 (in the presence of DTT) was electrophoresed on a $12 \%$ sodium dodecyl sulfate polyacrylamide gel electropheresis (SDS-PAGE) gel under denaturing conditions, and then transferred to a nitrocellulose membrane (Millipore, Billerica, MA, USA). The membrane was blocked with $5 \%$ bovine serum albumin (BSA; Sigma, St. Louis, MO, USA), washed and probed with mice sera at 1:50 for $1 \mathrm{~h}$ at $37^{\circ} \mathrm{C}$, followed by utilization of horseradish peroxidase (HRP)-conjugated goat anti-mouse IgG (Sigma) (Fig. 2A). The reaction was completed with $0.05 \%$ 3,3'-diaminobenzidine and $0.012 \% \mathrm{H}_{2} \mathrm{O}_{2}$ for $15 \mathrm{~min}$ at $37^{\circ} \mathrm{C}$.

Enzyme-linked immunosorbent assay (ELISA) for anti-HSP65 $\operatorname{IgG}$. Humoral immune responses to HSP65 were measured using an indirect ELISA as previously described (22). Briefly, 96-well flat-bottomed ELISA plates (Corning Costar, Acton, MA, USA) were coated with $100 \mu \mathrm{l} /$ well of HSP65 protein (50 $\mu \mathrm{g} /$ well) conjugated with $5 \% \mathrm{BSA}$ in $0.1 \mathrm{mM}$ carbonatebicarbonate buffer and stored overnight at $4^{\circ} \mathrm{C}$. Plates were blocked with PBS containing 5\% (w/v) BSA for $1 \mathrm{~h}$ and then incubated with $100 \mu \mathrm{l} /$ well 1:100 dilution of serum collected from the immunized animals in PBS containing 2\% BSA. Following incubation for $1 \mathrm{~h}$ at $37^{\circ} \mathrm{C}$, wells were washed with PBST (PBS containing 0.1\% Tween-20) three times, and then incubated with $100 \mu \mathrm{l} /$ well of HRP-conjugated goat antimouse IgG (Sigma) diluted at 1:20,000 in PBS containing 1\% BSA for $1 \mathrm{~h}$ at $37^{\circ} \mathrm{C}$. Wells were intensively washed 6 times in PBST and then incubated with $100 \mu \mathrm{l} /$ well peroxidase substrate $0.01 \%$ 3,3',5,5'-tetramethylbenzidine (TMB) and $0.24 \%(\mathrm{w} / \mathrm{v}) \mathrm{H}_{2} \mathrm{O}_{2}$-urea solubilized in $0.2 \mathrm{M} \mathrm{Na}_{2} \mathrm{HPO}_{4}$ to $0.1 \mathrm{M}$ citrate buffer $\left(\mathrm{pH} \mathrm{5.5)}\right.$ for $20 \mathrm{~min}$ at $37^{\circ} \mathrm{C}$. The reaction was terminated with $50 \mu \mathrm{l} /$ well of $2 \mathrm{M} \mathrm{H}_{2} \mathrm{SO}_{4}$ and finally the optical density 450 (OD450) value was measured using an ELISA reader (Bio-Rad, Hercules, CA, USA) (Fig. 2B). 
A

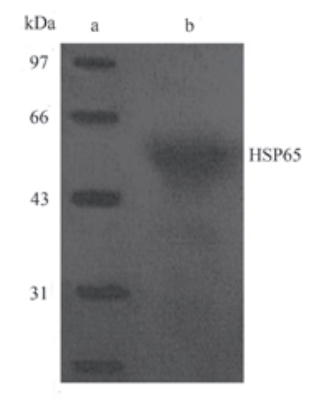

B

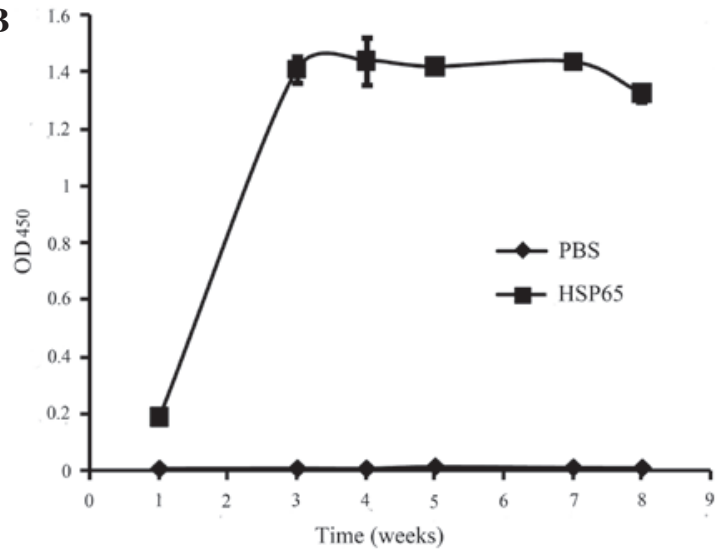

Figure 2. (A) Western blot analysis of specific anti-HSP65 antibodies. Lane a, protein molecular weight marker. Lane b, antibodies denoting HSP65. (B) ELISA analysis of anti-HSP65 IgG. Absorbance at $450 \mathrm{~nm}$ demonstrated antibody titers from the representative sera collected from the mice immunized with HSP65 and PBS. HSP65, heat shock protein 65; PBS, phosphate-buffered saline; OD, optical density. ELISA, enzyme-linked immunosorbent assay.

A

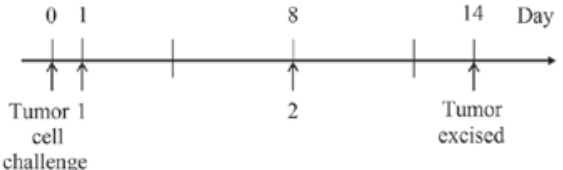

B

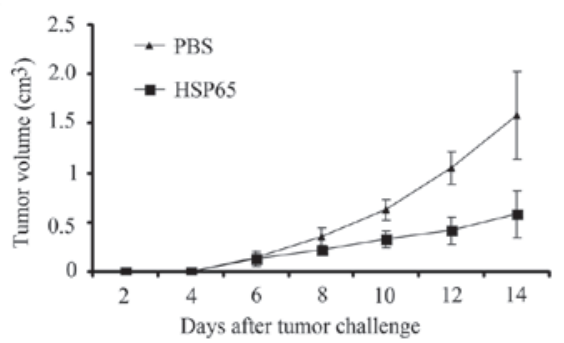

C

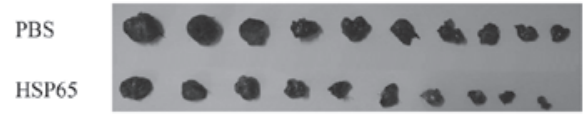

D

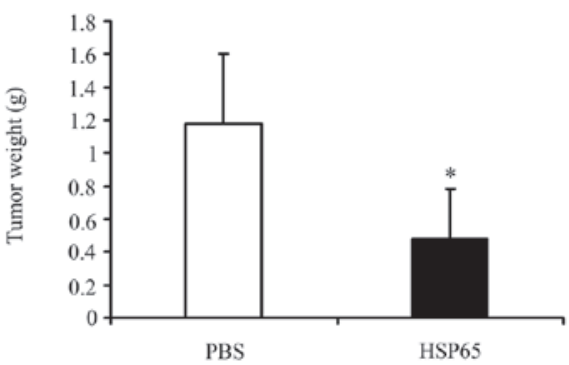

Figure 3. Therapeutic immunization of mice with vaccines. (A) Immunization scheme indicates the vaccination day as demonstrated by arrows. (B) In vivo measurement of the tumor growth. (C) Solid tumors were extracted from the mice immunized as described in A. (C) Tumor mass comparison between the mice immunized with HSP65 and PBS. "P<0.05. HSP65, heat shock protein 65; PBS, phosphate-buffered saline.

A

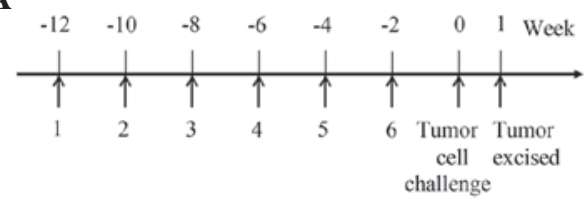

B
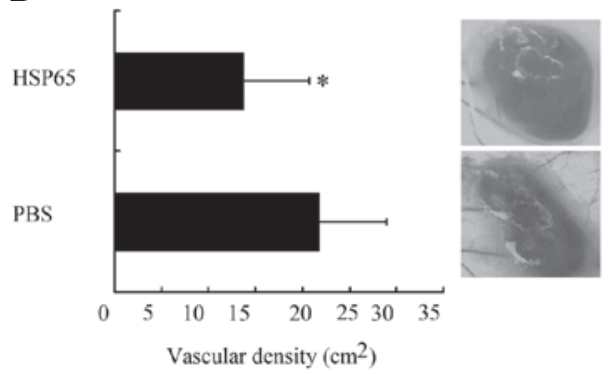

C

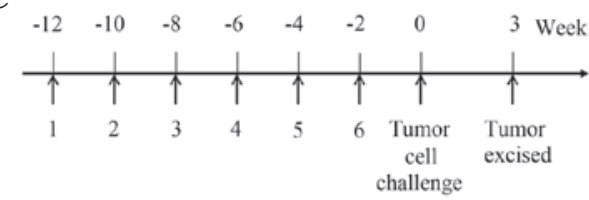

D

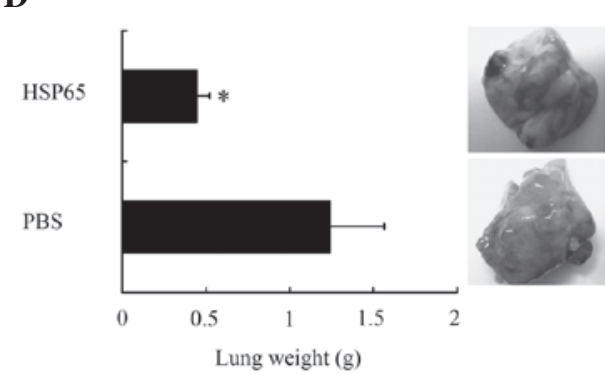

Figure 4. Effects of immune response on tumor-induced angiogenesis and pulmonary metastasis. (A) Immunization scheme of the tumor-induced angiogenesis experiment as demonstrated by arrows. (B) Total number of blood vessels (major vessels and branching points) was determined within a precise $1-\mathrm{cm}^{2}$ area around each implant site (2/mouse, 6 mice/group). Newly formed blood vessels were assessed by light microscopy (objective magnification, $\left.\mathrm{x} 1\right)$. (C) Immunization scheme of the tumor-induced pulmonary metastasis as demonstrated by arrows. (D) Lung mass comparison between the mice immunized with HSP65 and PBS. Lungs of mice (10 mice/group) were immunized as described in C. Values were expressed as the mean \pm SD. "P<0.05. HSP65, heat shock protein 65; PBS, phosphate-buffered saline. 
A

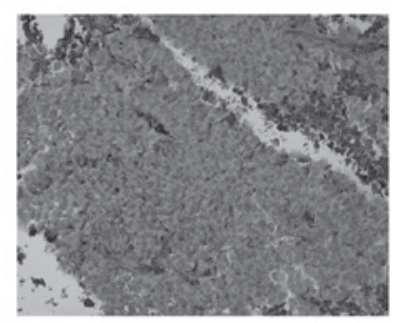

C

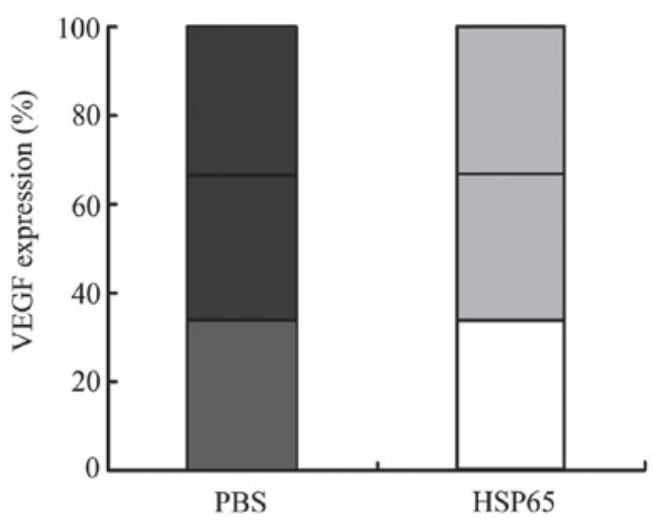

Figure 5. Immunohistochemical analysis of VEGF expression in tumors (magnification, $\mathrm{x} 100$ ). (A) H\&E staining photomicrograph of the pathological sections from the tumors of the PBS group. (B) H\&E staining photomicrograph of the pathological sections from the tumors of the HSP65 group (C) Comparison of VEGF expression between the mice immunized with PBS and HSP65. HSP65, heat shock protein 65; PBS, phosphate-buffered saline. $\mathrm{H} \& \mathrm{E}$, hemotoxylin and eosin; VEGF, vascular endothelial growth factor.

Tumor therapy efficacy of HSP65 protein in C57BL/6 mice. For tumor therapy experiments, two groups of C57BL/6 mice (10 per group) were injected subcutaneously into the right flank with $5 \times 10^{5}$ of B16-F10 cells per mouse on day 0 . The HSP65 group of mice were immunized subcutaneously into the left flank with the purified protein HSP65 (50 $\mu \mathrm{g}$ in a final volume of $100 \mu \mathrm{l}$ PBS) on day 1 . Booster injections were provided 1 week later subcutaneously in the left flank with purified protein HSP65 (Fig. 3A). The control group was administered an equal volume of blank PBS. Tumors were measured in two dimensions using a caliper every 2 days following the tumor challenge (Fig. 3B). All mice were sacrificed for tumor weight analysis 1 week after the challenge of tumor cells (Fig. 3C and D).

In vivo angiogenesis assay. To evaluate the effect of HSP65 on the neovascularization of the B16-F10 tumor, an intradermal tumor model was employed. In the model, neovasculature, discerned predominantly at the tumor periphery, was quantified using the vessel counting method as described by Kreisle (23). Briefly, 2 groups of mice (6 mice per group) were vaccinated with PBS or HSP65 6 times at biweekly intervals, respectively. An intracutaneous transplantation experiment was then performed by injecting $5 \times 10^{5}$ B16-F10 cells in $50 \mu 1$ of PBS into the mice at two sites in the abdominal region 2 weeks after the last immunization (Fig. 4A). On day 7 after the tumor implantation, all mice were sacrificed and the tumors were isolated for neovascularization analysis (Fig. 4B). For vessel counting, sections containing tumors were visualized using light microscopy (magnification, x10), and the total number of blood vessels (major vessels and branching points) within a $1-\mathrm{cm}^{2}$ area around each implant site was determined.

Experimental lung metastasis model. To evaluate the influence of HSP65 on the pulmonary metastasis of B16-F10 tumor, the tail intravenously-injected tumor model was used. Two groups of mice (10 each group) were vaccinated with PBS or HSP65 6 times at biweekly intervals, respectively. B16-F10 melanoma cells $\left(5 \times 10^{5}\right.$ cells/mouse) were injected into the tail veins of these mice 2 weeks after the last immunization (Fig. 4C). On day 21 after the challenge of tumor cells, all the mice were sacrificed for lung tumor analysis (Fig. 4D).

Statistical analysis. Data are expressed as the mean \pm standard deviation (SD). A Student's t-test was used to calculate the significance among the groups. $\mathrm{P}<0.05$ was considered to indicate a statistically significant difference.

\section{Results}

Western blot analysis of anti-HSP65 antibody. To determine the specificity of the antibodies from the mice immunized with HSP65, HSP65 was processed for western blot analysis using the antisera from mice vaccinated with HSP65. Antibodies representing HSP65 (lane 2) suggested that the antibodies specifically recognized the HSP65 antigen (Fig. 2A).

ELISA for anti-HSP65 IgG. In order to investigate whether the HSP65 protein vaccine was able to enhance the immunogenicity of the anti-HSP65 vaccine, an ELISA assay was conducted to determine the concentrations of anti-HSP65 antibodies in the sera collected from the mice immunized with HSP65 and PBS, respectively. HSP65-specific IgG antibody responses were evident in the mice immunized with HSP65, exhibiting a peak 3 weeks after the vaccination (Fig. 2B). In contrast, no antibody was elicited in the mice vaccinated with PBS. These observations indicate that HSP65 was capable of inducing an intense immune response.

Tumor protection efficacy of HSP65. To investigate whether HSP65 immunization was able to inhibit the growth of B16-F10 tumors in mice, a B16-F10 tumor model was utilized. Four weeks after the last immunization, half of the mice (10 per group) were sacrificed and the tumors were removed for tumor weight analysis. The growth of the tumor was monitored for 2 weeks (Fig. 1A). HSP65 immunization significantly inhibited the growth of B16-F10 tumor in mice (Fig. 1B). The mean tumor weight of the mice immunized with HSP65 was significantly lower than that of the PBS group $(\mathrm{P}<0.05$; Fig. 1C and D). In the survival monitoring experiment, all mice in the PBS group died, while $33.33 \%$ of the mice remained alive in the group immunized with HSP65 day 27 post-tumor implantation, displaying a prolonged survival. These results demonstrate that HSP65 effectively inhibited the growth of B16-F10 cells and prolonged the survival of mice (Fig. 1E).

Therapeutic efficacy of HSP65 on tumors. In order to determine the therapeutic effect of HSP65, two groups of C57BL/6 mice (10 mice per group) were inoculated with B16-F10 tumor cells $\left(5 \times 10^{5} /\right.$ mouse) on day 0 (Fig. $\left.3 \mathrm{~A}\right)$. The growth of the 
tumor was monitored for 2 weeks. In comparison with the PBS control group, tumor growth was significantly inhibited in the mice immunized with HSP65 (Fig. 3B). The mean tumor weight of the mice immunized with HSP65 was significantly lower than that of the PBS group $(\mathrm{P}<0.05$; Fig. $3 \mathrm{C}$ and $\mathrm{D})$.

Neovascularization inhibition. In order to evaluate the effect of HSP65 immunization on angiogenesis, a B16-F10 tumor model was applied. On day 7 after the challenge of tumor cells, all the mice were sacrificed for tumor analysis (Fig. 4A). For angiogenesis, the total number of blood vessels around each implant site from the mice immunized with HSP65 was significantly lower than that from the PBS group mice (Fig. 4B).

Pulmonary metastasis inhibition. The anti-metastatic effects of HSP65 were evaluated in the tail intravenously-injected model of mice inoculated with B16-F10 melanoma cells. On day 21 after the tumor cell challenge, all mice were sacrificed for lung tumor analysis (Fig. 4C). In the PBS group, three of the mice exhibited serious invasions of tumor cells in their lungs, which were completely surrounded by black tumors (data not shown). However, none of the HSP65 pretreated mice demonstrated the same phenomena, and the lung weights of the HSP65 group were much lower than those of the PBS group $(\mathrm{P}<0.05$; Fig. 4D).

Photomicrographs of the pathological slices of tumors. H\&E staining of the liver, lungs, heart, kidneys and spleen of the mice revealed no significant pathological changes between the HSP65 group and the PBS group (data not shown). Immunohistochemical analysis demonstrated that HSP65 immunization downregulated vascular endothelial growth factor (VEGF) expression in tumor tissues (Fig. 5).

\section{Discussion}

In this study, the antitumor effects and possible antitumor mechanism of HSP65 were explored. Mice were immunized with HSP65, and HSP65-specific antibodies were detected from the immunized mice sera. The growth, angiogenesis and metastasis of B16-F10 melanoma cells were significantly inhibited in vivo by the administration of HSP65, and the survival of vaccinated mice were prolonged as a result. VEGF expression was downregulated by HSP65 vaccination. To our knowledge, this is the first study to demonstrate that vaccination with the HSP65 protein inhibits the growth of B16-F10 murine melanoma cells in mice.

HSP60 is overexpressed in the majority of tumor cells (9-11,14-18). It has been demonstrated that HSP60 accumulates in cancer cells and is involved in their survival $(13,14)$. However, extracellular HSP60 mediates immunological functions to induce a specific antitumor immune response that is able to reject the tumor (19). HSPs have remained almost unchanged throughout evolution, and greater than $50 \%$ of HSP65 is identical to HSP60 (19,24-27). Owing to this homology, antibodies elicited against HSP65 are able to cross-react with HSP60 (28). Results from ELISA and western blot analysis verified that HSP65 was able to trigger effective immune responses, resulting in specific anti-HSP65 IgG antibodies (Fig. 2). The possible antitumor mechanism of HSP65 may be that the antibodies neutralized the elevated levels of HSP60 issued from the tumor cells, thereby blocking the activation of HSP60 in malignant melanoma cells.

Protein vaccination provides an attractive and acceptable strategy for cancer immunotherapy, accompanied by vaccine safety, cost-effectiveness and acceptance. A vaccination strategy against tumors may be considered successful after the demonstration of the inhibition of angiogenesis and metastasis of tumors.

Metastasis of tumor cells is difficult to approach in clinical therapeutics, as it induces cancer recurrence following surgery and may lead to the mortality of cancer patients. Malignant melanoma is among the most common causes accounting for 'metastatic cancer of unknown primary' (29), and has been well-documented as an angiogenic tumor $(30,31)$. Angiogenesis is critical for tumor growth and metastasis (2,2-34), and the use of anti-angiogenic agents is becoming feasible as the fourth cancer treatment following surgery, chemotherapy and radiotherapy (2).

Angiogenic signaling, which plays a critical role in melanoma, is mediated by pathways of growth factor receptors, including VEGF (35). VEGF is a glycosylated, multifunctional cytokine that is abundantly expressed and secreted by the majority of human and animal tumors. It is considered to play essential roles in the migration, proliferation and differentiation of endothelial cells in the tumor environment $(32,36,37)$, and in the stimulation of angiogenesis and tumor growth (37). VEGF has been revealed to be stimulated by intracellular HSP60 (38), and thus could be inhibited by extracellular HSP65/60 immunointervention, as observed in this study. Photomicrographs of pathological sections from tumors in this study demonstrate that VEGF expression in the tumors of HSP65-preimmunized mice were downregulated (Fig. 5), revealing that vaccination with HSP65 participated in the inhibition of tumor-induced angiogenesis and tumor growth (Fig. 4). As a result, the growth of the solid tumor of B16-F10 was remarkably inhibited by HSP65 vaccination (Figs. 1 and 3), which was demonstrated to be highly efficacious against B16-F10 tumors in vivo.

In conclusion, it has been preliminarily demonstrated that administration of the HSP65 protein is able to effectively inhibit the growth of B16-F10 melanoma in vivo. Further studies are required to investigate the anti-angiogenic mechanism of HSP65 and elucidate the underlying mechanism of HSP65 against melanoma.

\section{Acknowledgements}

This study was supported by the China National Natural Science Fund Committee (Grant nos. 30772570, 30672464, 30701023, 30500458 and 30872393), the Natural Science Foundation of Jiangsu Province (No. BK 2007170), and the Fundamental Research Funds for the Central Universities (Program nos. JKY2009021 and JKQ2009022).

\section{References}

1. Jemal A, Siegel R, Xu J and Ward E: Cancer statistics, 2010. CA Cancer J Clin 60: 277-300, 2010.

2. Emmett MS, Dewing D and Pritchard-Jones RO: Angiogenesis and melanoma - from basic science to clinical trials. Am J Cancer Res 1: 852-868, 2011. 
3. Carlson JA, Slominski A, Linette GP, et al: Malignant melanoma 2003: predisposition, diagnosis, prognosis, and staging. Am J Clin Pathol 120 Suppl: S101-S127, 2003.

4. Eggermont AM: Advances in systemic treatment of melanoma. Ann Oncol 21 Suppl 7: vii339-344, 2010.

5. Griguer CE, Oliva CR, Kelley EE, Giles GI, Lancaster JR Jr and Gillespie GY: Xanthine oxidase-dependent regulation of hypoxiainducible factor in cancer cells. Cancer Res 66: 2257-2263, 2006.

6. Schmitt E, Gehrmann M, Brunet M, Multhoff G and Garrido C: Intracellular and extracellular functions of heat shock proteins: repercussions in cancer therapy. J Leukoc Biol 81: 15-27, 2007.

7. Graner MW, Cumming RI and Bigner DD: The heat shock response and chaperones/heat shock proteins in brain tumors: surface expression, release, and possible immune consequences J Neurosci 27: 11214-11227, 2007.

8. Eustace BK and Jay DG: Extracellular roles for the molecular chaperone, hsp90. Cell Cycle 3: 1098-1100, 2004.

9. Xu X, Wang W, Shao W, et al: Heat shock protein-60 expression was significantly correlated with the prognosis of lung adenocarcinoma. J Surg Oncol 104: 598-603, 2011.

10. Lin C-S, He P-J, Tsai N-M, et al: A potential role for Helicobacter pylori heat shock protein 60 in gastric tumorigenesis. Biochem Biophys Res Commun 392: 183-189, 2010.

11. Hwang YJ, Lee SP, Kim SY, et al: Expression of heat shock protein $60 \mathrm{kDa}$ is upregulated in cervical cancer. Yonsei Med J 50: 399-406, 2009.

12. Cappello F, David S, Rappa F, et al: The expression of HSP60 and HSP10 in large bowel carcinomas with lymph node metastase. BMC Cancer 5: 139, 2005.

13. Kamal A, Boehm MF and Burrows FJ: Therapeutic and diagnostic implications of Hsp90 activation. Trends Mol Med 10: 283-290, 2004

14. Garrido C, Schmitt E, Cande C, Vahsen N, Parcellier A and Kroemer G: HSP27 and HSP70: potentially oncogenic apoptosis inhibitors. Cell Cycle 2: 579-584, 2003.

15. Sedlackova L, Spacek M, Holler E, Imryskova $Z$ and Hromadnikova I: Heat-shock protein expression in leukemia. Tumour Biol 32: 33-44, 2010.

16. Xiong Q, Jin L, Li J, et al: A Th2 immune shift to heat shock protein 65 fails to arrest atherosclerosis: proatherogenic role of Th2-deviated autoantibodies. Autoimmunity 42: 475-483, 2009.

17. Ausiello CM, Fedele G, Palazzo R, Spensieri F, Ciervo A and Cassone A: $60-\mathrm{kDa}$ heat shock protein of Chlamydia pneumoniae promotes a $\mathrm{T}$ helper type 1 immune response through IL-12/IL-23 production in monocyte-derived dendritic cells. Microbes Infect 8: 714-720, 2006.

18. Chen K, Lu J, Wang L and Gan YH: Mycobacterial heat shock protein 65 enhances antigen cross-presentation in dendritic cells independent of Toll-like receptor 4 signaling. J Leukoc Biol 75: 260-266, 2004

19. Zhao Y, Yokota K, Ayada K, et al: Helicobacter pylori heat-shock protein 60 induces interleukin-8 via a Toll-like receptor (TLR)2 and mitogen-activated protein (MAP) kinase pathway in human monocytes. J Med Microbiol 56: 154-164, 2007.

20. Jin L, Wang Y, Xiong Q, et al: Long-lasting specific antibodies against P277 induced by mucosal administration of P277 repeat sequences carried by Hsp65 in the absence of adjuvants. Vaccine 25: 2043-2050, 2007.
21. Lu Y, Ouyang K, Fang J, et al: Improved efficacy of DNA vaccination against prostate carcinoma by boosting with recombinant protein vaccine and by introduction of a novel adjuvant epitope. Vaccine 27: 5411-5418, 2009

22. Guojun W, Wei G, Kedong O, et al: A novel vaccine targeting gastrin-releasing peptide: efficient inhibition of breast cancer growth in vivo. Endocr Relat Cancer 15: 149-159, 2008.

23. Kreisle RA and Ershler WB: Investigation of tumor angiogenesis in an id mouse model: role of host-tumor interactions. J Natl Cancer Inst 80: 849-854, 1988.

24. Xiong Q, Li J, Jin L, Liu J and Li T: Nasal immunization with heat shock protein 65 attenuates atherosclerosis and reduces serum lipids in cholesterol-fed wild-type rabbits probably through different mechanisms. Immunol Lett 125: 40-45, 2009.

25. Marcatili A, Cipollaro de l'Ero G, Galdiero M, Folgore A and Petrillo G: TNF-alpha, IL-1 alpha, IL-6 and ICAM-1 expression in human keratinocytes stimulated in vitro with Escherichia coli heat-shock proteins. Microbiology 143 (Pt 1): 45-53, 1997.

26. Lillicrap MS, Duggleby RC, Goodall JC and Gaston JS: T cell recognition of a highly conserved epitope in heat shock protein 60: self-tolerance maintained by TCR distinguishing between asparagine and aspartic acid. Int Immunol 16: 405-414, 2004.

27. Hartl FU and Hayer-Hartl M: Molecular chaperones in the cytosol: from nascent chain to folded protein. Science 295: 1852-1858, 2002

28. Verdegaal ME, Zegveld ST and van Furth R: Heat shock protein 65 induces CD62e, CD106, and CD54 on cultured human endothelial cells and increases their adhesiveness for monocytes and granulocytes. J Immunol 157: 369-376, 1996.

29. Chin L, Garraway LA and Fisher DE: Malignant melanoma: genetics and therapeutics in the genomic era. Genes Dev 20: 2149-2182, 2006.

30. Ferrara N: Role of myeloid cells in vascular endothelial growth factor-independent tumor angiogenesis. Curr Opin Hematol 17: 219-224, 2010

31. Shojaei F, Zhong C, Wu X, Yu L and Ferrara N: Role of myeloid cells in tumor angiogenesis and growth. Trends Cell Biol 18: 372-378, 2008

32. Yang L, Chen G, Mohanty S, et al: GPR56 regulates VEGF production and angiogenesis during melanoma progression. Cancer Res 71: 5558-5568, 2011.

33. Folkman $\mathrm{J}$ : What is the evidence that tumors are angiogenesis dependent? J Natl Cancer Inst 82: 4-6, 1990.

34. Carmeliet P and Jain RK: Angiogenesis in cancer and other diseases. Nature 407: 249-257, 2000.

35. Sekulic A, Haluska P Jr, Miller AJ, et al: Malignant melanoma in the 21 st century: the emerging molecular landscape. Mayo Clin Proc 83: 825-846, 2008

36. Carmeliet P: VEGF as a key mediator of angiogenesis in cancer. Oncology 69 Suppl 3: 4-10, 2005.

37. Verheul HM, Hammers H, van Erp K, et al: Vascular endothelial growth factor trap blocks tumor growth, metastasis formation, and vascular leakage in an orthotopic murine renal cell cancer model. Clin Cancer Res 13: 4201-4208, 2007.

38. Pugh CW and Ratcliffe PJ: Regulation of angiogenesis by hypoxia: role of the HIF system. Nat Med 9: 677-684, 2003. 